\title{
Single bacterial cell mass analysis: a rapid test method in leprosy therapy control
}

\author{
U SEYDEL \& B LINDNER \\ Forschungsinstitut Borstel, Division of Biophysics, 2061 Borstel, \\ $F R G$
}

\section{Introduction}

A major characteristic of Hansen's disease distinguishing it from other bacterial infections is the fact that it is caused by a bacterial species not yet satisfactorily cultivable in vitro. Despite the severe problems which were and still are brought about by this fact, successful treatments of the disease, particularly the multidrug regime introduced by Freerksen $\&$ Rosenfeld ${ }^{1}$ could be developed. Nevertheless, there remain several questions to be answered concerning: (i) the kinetics of the bacterial in vivo response to the chemotherapy - being associated with the dosage of the drugs; (ii) criteria for the duration of treatment; and (iii) the occurrence of drug resistance and persisters. For these reasons strong efforts have been made towards the development of techniques for the rapid detection of bacterial cell impairment and for in vitro drug screening.

Here, the groups of A M Dhople and P R Mahadevan should be mentioned in particular as they have developed in vivo systems for monitoring drug response of Mycobacterium leprae which are based either on an ATP-assay ${ }^{2}$ or on EA rosetting of macrophages, ${ }^{3}$ both applied to human biopsy material. Furthermore, both groups have extended their systems to in vitro drug screening by introducing artificial nutrients which not only keep host-derived $M$. leprae alive for several weeks but also allow for their limited multiplication. ${ }^{4,5}$ We have described an in vivo test system based on the single bacterial cell mass analytical determination of the intracellular $\mathrm{Na}^{+}, \mathrm{K}^{+}$-ratio, ${ }^{6,7}$ which we have just started to extend for invitro drug screening.

This contribution will first give a short introduction to the general features of the technique, combined with a review of some already published results on human biopsy material and, secondly, the presentation of some very recent data from in vivo studies of drug kinetics and in vitro studies of drug action on armadillo-derived $M$. leprae in artificial media. 


\section{Principles of the method and review}

In Figure 1 the general procedure is outlined schematically. The bacteria are prepared on Formvar-filmed copper meshes (as used in electron microscopy) either from cell cultures or -in the case of the non-cultivable $M$. leprae-from biopsies via isolation from tissue, usually according to the protocol of Dhople ${ }^{8}$ (see top). In each case the bacteria are washed quickly twice in distilled water to

\section{sample}
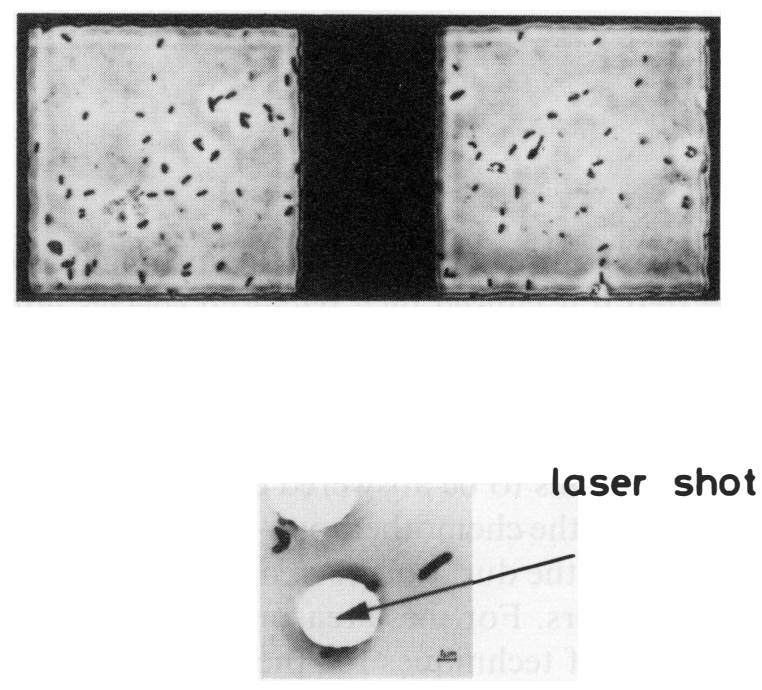

mass spectra
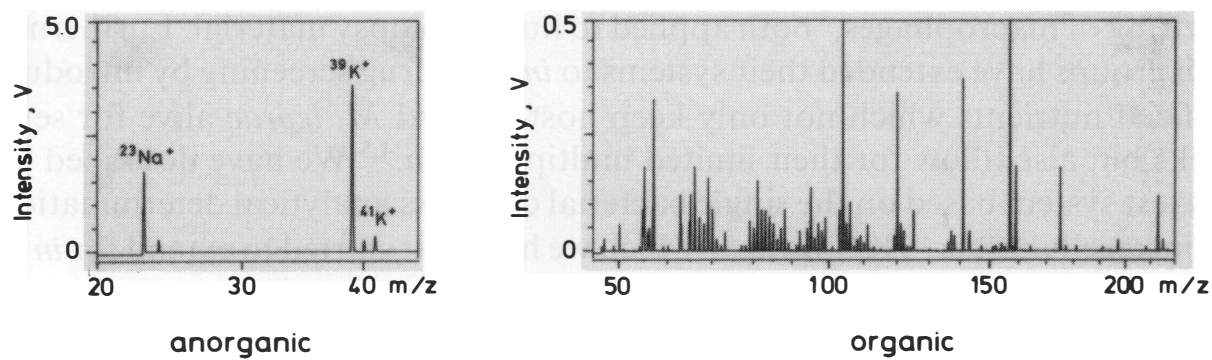

Figure 1. Schematic of single bacterial cell mass analytical procedure. Top: bacteria prepared on Formvar-filmed Cu-mesh (detail). Middle: laser-burnt hole in Formvar-film af ter vaporization of a bacterial cell (enlargement). Bottom: mass spectrometric information from a single cell on cation concentrations (left) or on organic fragment ions (right). 
avoid contamination. The mesh is then mounted within a laser microprobe mass analyser LAMMA 500 (Leybold-Heraeus, Köln, West Germany).

A single cell at a time is vaporized, ionized, and subsequently mass analysed upon the irradiation with a high-power laser pulse (see Figure 1 middle). The produced so-called laser desorption mass spectra cover as well direct information on the intracellular cation concentrations of a single cell (see Figure 1 bottom, left) as indirect information on its organic matrix (see Figure 1 bottom, right). For a complete evaluation of a sample typically a few hundred cells are analysed this way, requiring only some hours. In first experiments with cultivable bacteria, mainly E. coli, we have shown that the ratio of the intracellular $\mathrm{Na}^{+}$and $\mathrm{K}^{+}$ concentrations is a sensitive indicator of the physiological state of a cell and its alterations.

For this we have treated $E$. coli cultures with a chemotherapeutic and followed changes in the $\mathrm{Na}^{+}, \mathrm{K}^{+}$-ratio from 30 single cells prepared at different times af ter drug application. These results were compared with various other data like total cell number, ${ }^{14} \mathrm{CO}_{2}$-production, number of colony forming units, and ATPrelease and were found to be in excellent agreement with these. ${ }^{6}$ As an example, the single cell mass spectra of a viable untreated (left) and of a treated cell (right), here $M$. leprae, showing the drastic changes in the $\mathrm{Na}^{+}$and $\mathrm{K}^{+}$signals, are given in Figure 2.

Figure 3 shows, for the experiment described above, the comparison between single cell and the CFU-data.

A special feature of the single cell mass analytical technique is the possibility of establishing distributions of the $\mathrm{Na}^{+}, \mathrm{K}^{+}$-ratio within a larger number of
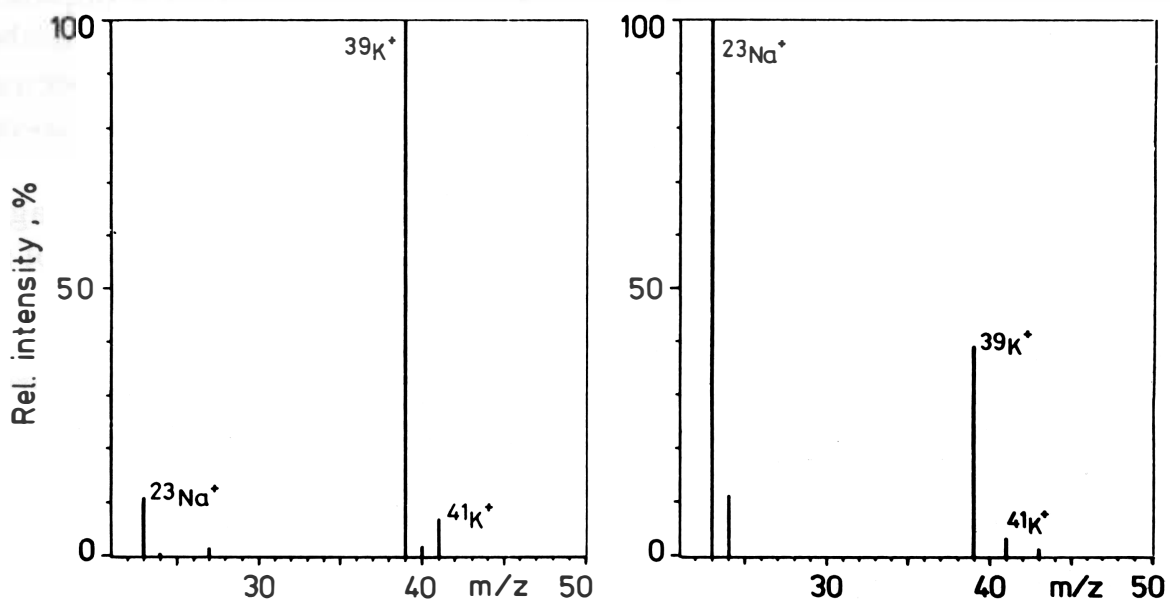

Figure 2. Cation signals from single $M$. leprae cells isolated from skin biopsies of a patient before (left) and 4 months after the beginning of DDS-monotherapy (right). 


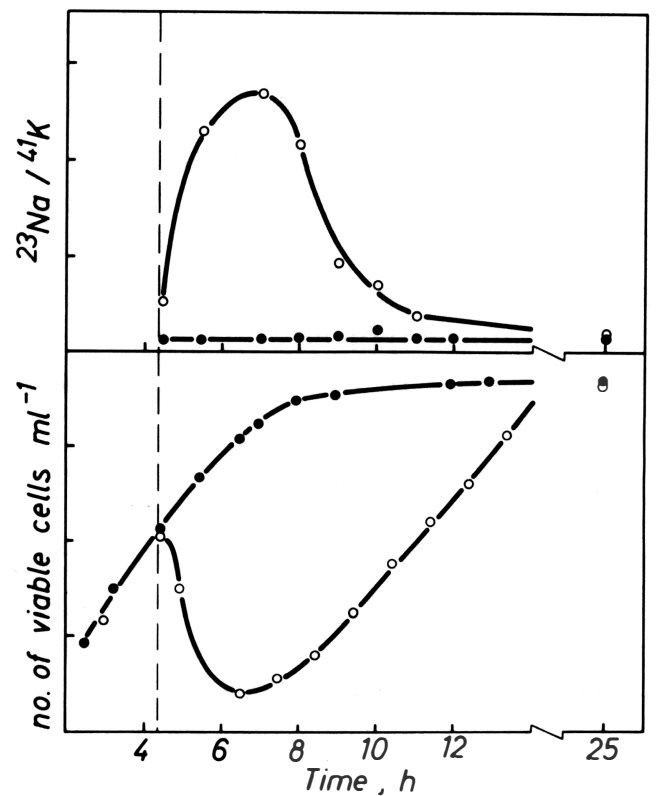

Figure 3. Time dependent responses of E. coli to treatment with the nitrofuran derivative HN32 at 5 fold the MIC. Top: ${ }^{23} \mathrm{Na}^{+},{ }^{41} \mathrm{~K}^{+}$-ratio averaged over 30 single cells from each measuring point. Bottom: viable cell number from plate counts. The drug was added at the time shown by the vertical dashed line.

single cells and this way having insight into the viability within a population. From this it should be possible to get well-founded information on the number of viable cells in isolated $M$. leprae. As mentioned above, the organic cell spectra can provide additional, but indirect information when they are treated as so-called mass fingerprints. Of course, the organic matrix of a bacterial cell is very complex and the interaction of the laser with the matrix leads to considerable fragmentation of the mostly very large biomolecules and thus to a larger number of small ions. These ions are registered as mass peaks in the spectra. Due to the unknown fragmentation pathways it is impossible - at least at the moment - to assign these mass peaks to particular molecules or functional groups. However, as mentioned already, the entity of all peaks can serve as mass fingerprints which are characteristic for a given bacterial sample. Applying sophisticated computeraided mathematical multivariate cluster analytical procedures it is possible to obtain numerical relationships on the basis of which a separation between the various samples - either between different bacterial species or even between treated and untreated $M$. leprae cells isolated from human skin biopsies ${ }^{7,9}$-is possible. 


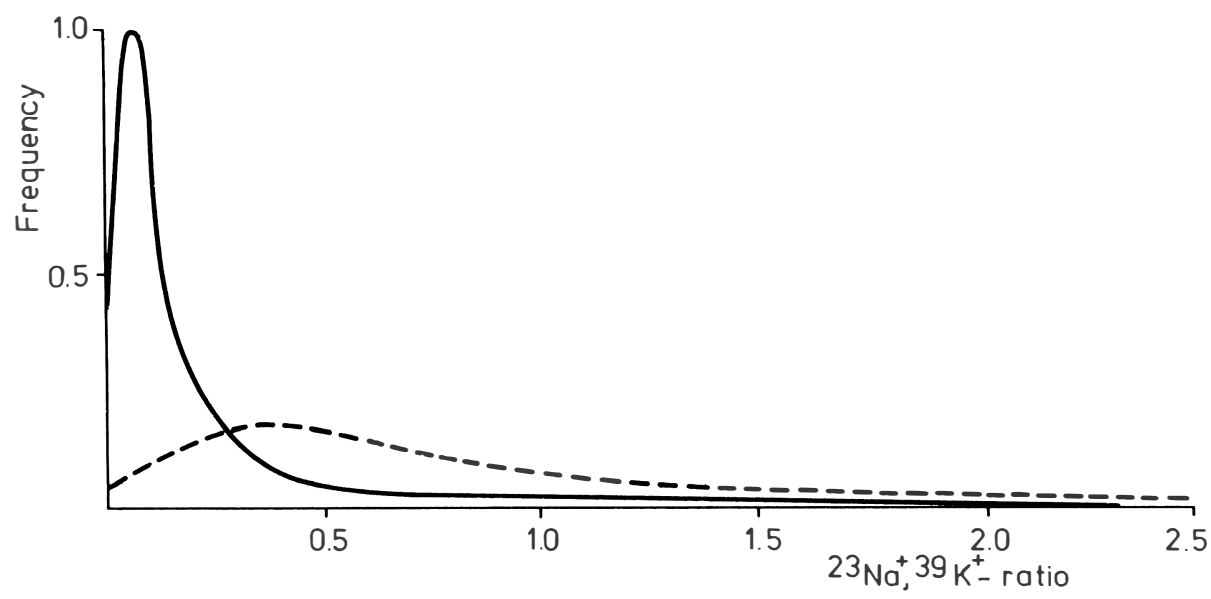

Figure 4. Distribution of the intracellular ${ }^{23} \mathrm{Na}^{+},{ }^{39} \mathrm{~K}^{+}$-ratios for two $M$. leprae populations of 400 cells each isolated from the same patient before (- - , and 4 months after $(---)$ the beginning of DDS therapy.

The applicability of the above outlined single cell technique to human-derived M. leprae could be demonstrated in an extensive study. ${ }^{7}$ It could be shown that, in spite of the relatively tough isolation procedure, distributions of the intracellular $\mathrm{Na}^{+}, \mathrm{K}^{+}$-ratio within a population of 400 single cells isolated from the same patient before and 4 months after the beginning of DDS-monotherapy could be obtained, which resembles the findings from cultivable species (Figure 4). Furthermore, for about 40 patient biopsies - either untreated or under DDSmonotherapy for different periods of time- a good agreement between the results from intracellular $\mathrm{Na}^{+}, \mathrm{K}^{+}$-ratios and those from the ATP-assay (and, where performed, also those from mouse footpad test) could be stated.

Interestingly, from a combination of the cation measurements and the fingerprint evaluation it was suggested in two cases, that the bacteria were DDSresistant. This was, in fact, later on confirmed by mouse footpad tests.

\section{Actual results and discussion}

The response to DDS-monotherapy as determined from changes in the intracellular $\mathrm{Na}^{+}, \mathrm{K}^{+}$-ratio of 88 human biopsy derived $M$. leprae samples after different periods of treatment - the preparations were kindly provided by A $\mathbf{M}$ Dhople - in dependence on the time of treatment, is demonstrated in Figure 5, showing a continuous increase until about 3-4 months after initiation of the therapy with no significant changes afterwards. This result represents the in vivo kinetics of the cell impairment due to drug action. From each sample-between 


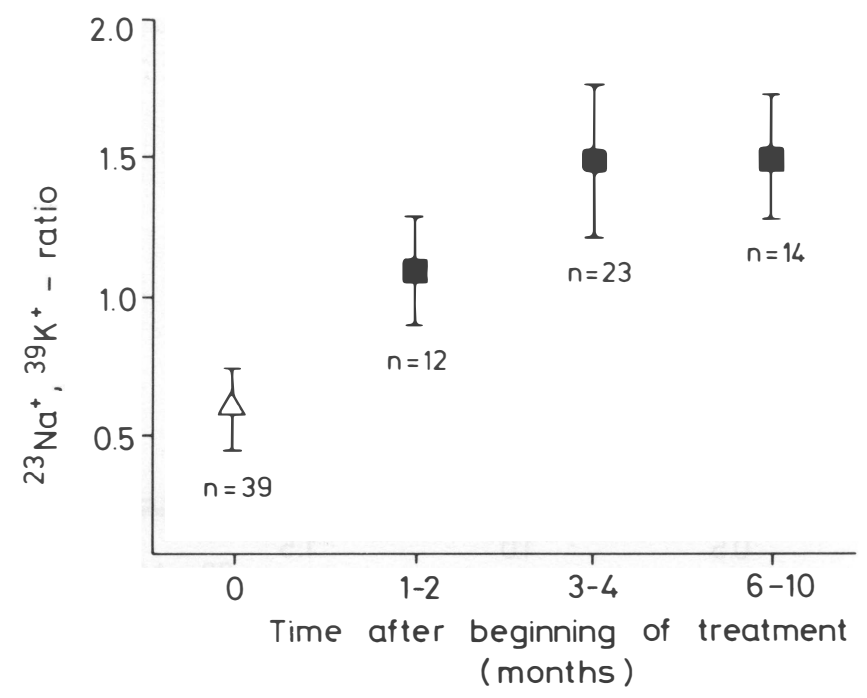

Figure 5. Time dependence of in vivo response of $M$. leprae to DDS therapy determined from the measurement of their intracellular ${ }^{23} \mathrm{Na}^{+},{ }^{39} \mathrm{~K}^{+}$-ratios after isolation from human skin biopsies. $(n$ gives the number of patients included in each measuring point.) From each biopsy 100 single cells were analysed and the cation ratios averaged.

12 and 39 for the different periods of treatment - the average over 100 single cell values was taken. Even though this evaluation is very rough, especially according to the length of the measuring intervals, it may demonstrate the capacity of this technique for obtaining information on different drug combinations from in vivo studies. It may, furthermore, supply data for an optimal dosage (concentration and application intervals).

In a first series of experiments we have tested the applicability of the single cell method to in vitro drug screening. For this, aliquots of $M$. leprae isolated from armadillo liver were incubated in Middlebrook $7 \mathrm{H} 9$ growth medium at a concentration of approximately $10^{9} \mathrm{ml}^{-1}$. To one batch DDS was added at a concentration of $5 \mu \mathrm{g} \mathrm{ml}^{-1}$. After $24 \mathrm{hr}$ the bacteria were prepared for cation determination.

Figure 6 gives the relative cumulative distributions of the $\mathrm{Na}^{+}, \mathrm{K}^{+}$-ratio within a population of 600 single cells in each case for bacteria analysed directly after the isolation from the liver (A), after inoculation in 7H9 medium (B), and after inoculation in medium + DDS (C). These results are compared with respective data from $M$. smegmatis also grown in $7 \mathrm{H} 9$ and harvested at the end of the exponential growth phase (D). From the relative cumulative distribution it can be deduced how many percent of the analysed bacteria have a $\mathrm{Na}^{+}, \mathrm{K}^{+}$-ratio smaller than a given value. Thus, for example, from the $M$. smegmatis sample about $90 \%$ of the analysed cells have a $\mathrm{Na}^{+}, \mathrm{K}^{+}$-ratio below 1 , whereas from the 


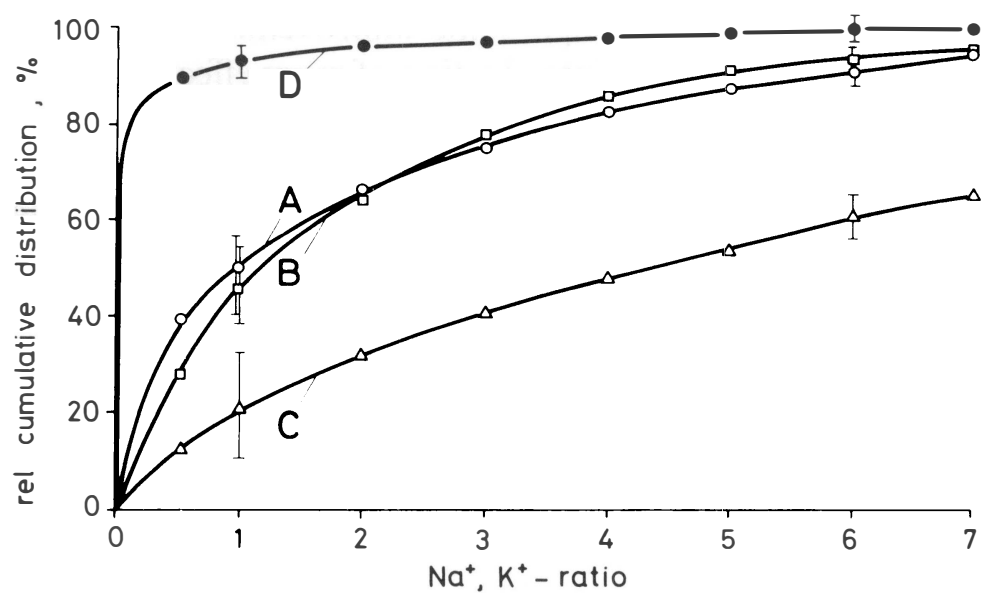

Figure 6. First results on in vitro drug screening showing the relative cumulative distributions of the ${ }^{23} \mathrm{Na}^{+},{ }^{39} \mathrm{~K}^{+}$-ratios within populations of 600 single cells in each case for: A, M. leprae isolated from armadillo liver; B, as A but after inoculation in Middlebrook medium $7 \mathrm{H} 9$ for $24 \mathrm{hr}$; C, as B but under action of $5 \mu \mathrm{g} \mathrm{ml}^{-1}$ DDS; and D, M. smegmatis grown in 7H9 and harvested at the end of exponential growth.

directly isolated $M$. leprae only about $45 \%$ have values below 1 . For the DDStreated $M$. leprae this figure decreases to about $20 \%$.

Taking a $\mathrm{Na}^{+}, \mathrm{K}^{+}$-ratio of 0.6 as an average value for untreated, unimpaired $M$. leprae cells from skin biopsies (see also Figure 5) it may be concluded from Figure 6 that about $30 \%$ of the bacteria directly analysed after isolation from armadillo liver show this or an even lower value. This percentage $(30 \%)$ is considerably higher than that of $0 \cdot 1 \%$ given in the literature and based on transfer experiments to mouse footpads. Thus, it may be assumed, that the low percentage of multiplying cells in mouse footpads cannot be explained by a low number of $a$ priori viable cells in the isolate but by adaptation problems to the new environment.

The discussion so far shows that the single cell mass analysis offers a possibility for differentiating between viable and impaired dead cells within a population. Curve $\mathrm{C}$ shows the influence of a drug (DDS, $5 \mu \mathrm{g} \mathrm{ml}^{-1}$ ) on the $\mathrm{Na}^{+}$, $\mathrm{K}^{+}$-ratio of the armadillo derived $M$. leprae after $24 \mathrm{hr}$. Even when considering that the medium used (Middlebrook 7H9) does not support multiplication-but, as can be taken from curve $\mathrm{C}$ also does not affect the bacterial viability negatively within the observed interval of $24 \mathrm{hr}$ - the drug action on the physiological state of the bacteria can clearly be derived. Of course, the drug concentration in these first measurements (each curve represents the average over three independent experiments) was comparatively high. However, we wanted to find out in this first step the general applicability of our technique for in vitro drug screening. This can 
be answered positively, even though some improvements may be considered, concerning, in the first place, the introduction of more effective growth media as developed for instance by Dhople ${ }^{4}$ and Mahadevan ${ }^{5}$ and their coworkers. Nevertheless, it should be kept in mind that the aim in improving this method is the development of fast data acquisition, that means within a few days.

\section{Acknowledgment}

We gratefully acknowledge the financial support of the German Leprosy Relief Association and the skilful technical assistance of Mrs G Dethlefs-Bubritzki.

\section{References}

1 Freerksen E, Rosenfeld M. Drug Res, 1972; 22: 1235-1242.

${ }^{2}$ Dhople AM, Hanks JH. Int J Lepr, 1981; 49: 57-59.

${ }^{3}$ Mankar MV, Birdli TJ, Mahadevan PR, Antia NH. Proc. of the 12th Int. Leprosy Congr., New Delhi, Febr. 20-25 (1984) pp. 706-707.

${ }^{4}$ Dhople AM. Lepr Rev (this volume).

${ }^{5}$ Mahadevan PR. Lepr Rev (this volume).

${ }^{6}$ Lindner B, Seydel U. J Gen Microbiol, 1983; 129: 51-55.

7 Seydel U, Lindner B, Dhople AM. Int J Lepr, 1985; 53: 365-375.

${ }^{8}$ Dhople AM, Storrs EE. Int J Lepr, 1982; 50: 83-89.

${ }^{9}$ Lindner B, Seydel U. J Phys Colloque (Paris) 1984; 45(C2): 565-568. 